\title{
Efeitos da pandemia da COVID-19 e suas repercussões no desenvolvimento infantil:
}

\section{Uma revisão integrativa}

\author{
Effects of the COVID-19 pandemic and its repercussions on child development: An integrative \\ review \\ Efectos de la pandemia del COVID-19 y sus repercusiones en el desarrollo infantil: Una revisión \\ integradora
}

\section{Resumo}

A pandemia pode ter afetado a saúde mental e impactado na rotina das crianças do mundo inteiro. Este estudo objetiva compreender como as crianças estão vivenciando os impactos da pandemia e as suas repercussões no desenvolvimento infantil. Realizou-se uma revisão integrativa da literatura nacional e internacional. A pesquisa foi realizada nos meses de março e abril do ano de 2020, nas bases de dados Pepsic, Scielo e Google Acadêmico. A amostra final foi composta por 12 estudos, estes foram agrupados em quatro categorias temáticas: (1) Tecnologia e saúde mental envolvendo as crianças durante a pandemia da COVID-19; (2) A importância dos pais falarem sobre o processo de morte e morrer com os filhos; (3) A violência infantil no contexto da COVID-19 e (4) Informações para manter a saúde mental das crianças. Verificou-se uso exacerbado das tecnologias, dificuldades dos cuidadores em dialogar com os filhos sobre o processo de morte, bem como aumento de problemas de comportamento, da violência infantil em crianças. Conclui-se que há necessidade de intervenções preventivas que ampliem as estratégias de enfrentamento dos cuidadores de crianças, para minimizar os prejuízos na saúde mental dos pequenos e do grupo familiar durante ou após a pandemia.

Palavras-chave: Saúde mental; Cuidadores; Criança; COVID-19; Psicologia.

\begin{abstract}
The pandemic may have affected the mental health and impacted the routine of children around the world. This study aims to understand how children are experiencing the impacts of the pandemic and its repercussions on child development. An integrative review of national and international literature was conducted. The research was conducted in the months of March and April 2020, in the Pepsic, Scielo, and Google Academic databases. The final sample was composed of 12 studies, these were grouped into four thematic categories: (1) Technology and mental health involving children during the COVID-19 pandemic; (2) The importance of parents talking about the process of death and dying with their children; (3) Childhood violence in the context of COVID-19 and (4) Information to maintain children's mental health. It was verified exacerbated use of technologies, difficulties of caregivers in dialoguing with their children about the process of death, as well as an increase in behavioral problems, child violence in children. We conclude that there is a need for preventive interventions to expand the coping strategies of child caregivers in order to minimize the damage to the mental health of the children and the family group during or after the pandemic.
\end{abstract}

Keywords: Mental Health; Caregivers; Child; COVID-19; Psychology. 


\section{Resumen}

La pandemia puede haber afectado a la salud mental y repercutido en la rutina de los niños de todo el mundo. Este estudio pretende comprender cómo los niños están experimentando los impactos de la pandemia y sus repercusiones en el desarrollo infantil. Se realizó una revisión integradora de la literatura nacional e internacional. La investigación se realizó en marzo y abril de 2020, en las bases de datos Pepsic, Scielo y Google Acadêmico. La muestra final estaba compuesta por 12 estudios, que se agruparon en cuatro categorías temáticas: (1) La tecnología y la salud mental de los niños durante la pandemia de COVID-19; (2) La importancia de que los padres hablen con sus hijos sobre el proceso de la muerte y la agonía; (3) La violencia infantil en el contexto de COVID-19 y (4) La información para mantener la salud mental de los niños. Se comprobó el uso exacerbado de las tecnologías, las dificultades de los cuidadores para dialogar con los niños sobre el proceso de la muerte, así como el aumento de los problemas de comportamiento y la violencia infantil en los niños. Se concluye que se necesitan intervenciones preventivas que amplíen las estrategias de enfrentamiento de los cuidadores de niños, para minimizar los perjuicios en la salud mental de los niños y del grupo familiar durante o después de la pandemia.

Palabras clave: Salud mental; Cuidadores; Niño; COVID-19; Psicología.

\section{Introdução}

A COVID-19 é uma doença causada pelo vírus $S A R S$-CoV-2 (sigla do inglês que significa coronavírus 2 da síndrome respiratória aguda grave). O indivíduo poderá apresentar quadro de infecção assintomática ou sintomas respiratórios mais graves. Esta doença possui uma alta transmissão entre as pessoas e tem como sintomas comuns a febre, a tosse e o cansaço (OPAS, 2020).

O distanciamento social pode ser experienciado negativamente pelas famílias e crianças, devido à quebra de rotina e a incerteza de quando a vida voltará à normalidade. Uma das principais mudanças na rotina refere-se à ausência das aulas presenciais das crianças e estas manifestam saudades dos professores e colegas, assim como das trocas que o ambiente escolar proporciona, tanto no processo de ensino aprendizagem como nas relações sociais (Fiocruz, 2020a).

Ao analisar o panorama das pessoas infectadas e doentes pela COVID-19, os pesquisadores percebem que as crianças são mais resistentes e seus quadros menos graves se comparadas com pessoas adultas e idosas (Zimmermann \& Curtis, 2020). Entretanto, estão sujeitas tanto quanto os adultos, ao estresse, ao medo de infecção, à ansiedade por não terem uma resposta referente à volta da normalidade de sua rotina. Ademais, outros sintomas que podem afetar a saúde mental de crianças e adultos estão sendo estudados, como a solidão, desesperança, exaustão, irritabilidade e a sensação de abandono (Fiocruz, 2020a).

Esses sintomas psicológicos são desencadeados devido à pandemia da COVID-19, ser um território desconhecido e ameaçador não só para as crianças, como também para os adultos. Esses sentimentos de inseguranças e incerteza, medo e desespero são intensificados quando as crianças são impossibilitadas de estarem com seus pares na escola, de saírem para brincar na pracinha, quando distanciadas dos avós e muitas vezes sofrem devido às restrições financeiras dos pais (SBP, 2020). Outro fator que pode acabar refletindo na saúde mental das crianças é a solidão devido os pais se ausentaram na modalidade de trabalho home office. O suporte social minimiza esses efeitos devastadores do sentimento de solidão e a possibilidade de troca ajuda a manter a saúde psíquica (Rodrigues, 2018).

A rotina pode ser considerada um fator protetivo quanto aos sentimentos de solidão, estresse e ansiedade. É fundamental que a criança compreenda que não está em um período de férias e dessa forma dê continuidade nas tarefas da escola. Além disso, as tarefas domésticas podem ser utilizadas tanto para o aprendizado quanto para o ensino de responsabilidades, sempre respeitando a idade e limites da criança. A tecnologia também surge como um fator positivo, pois minimiza o distanciamento dos amigos e familiares, bem como serve como uma ferramenta de aprendizado e diversão. No entanto, é necessário um cuidado por parte dos pais e responsáveis pelo conteúdo que a criança acessa (Fiocruz, 2020a).Assim, objetivou-se compreender como as crianças estão vivenciando os impactos da pandemia e as suas repercussões no desenvolvimento infantil. 


\section{Método}

Trata-se de um estudo de revisão integrativa da literatura sobre como tem sido evidenciado nas pesquisas os conteúdos que abordam sobre os impactos à saúde mental das crianças e os efeitos do cenário pandêmico para o desenvolvimento. A revisão integrativa seguiu cinco etapas fundamentais: (1) formulação do problema, por meio de uma pergunta norteadora; (2) busca dos materiais nas bases de dados; (3) critérios de inclusão/exclusão dos conteúdos; (4) análise e classificação dos materiais achados; (5) apresentação dos resultados encontrados (Silva et al., 2020).

A pergunta norteadora buscou compreender "Quais são os impactos e as repercussões da pandemia para o desenvolvimento infantil?”. A questão de pesquisa foi elaborada por meio da estratégia PICO que auxiliou na busca da melhor informação científica disponível. Seus componentes foram decompostos em P (Paciente), I (Interesse), C (Contexto) e O (Desfecho) Onde P é (crianças), I (impactos e as repercussões), C (pandemia) e O (desenvolvimento infantil).

A pesquisa foi realizada nos meses de março e abril do ano de 2020. Utilizaram-se as bases de dados on-line Pepsic, Scielo e Google Acadêmico para o levantamento de dados. Foram utilizados os operadores booleanos OR e AND, para facilitar o processo de busca. Usaram-se as seguintes palavras-chave: Saúde Mental; Desenvolvimento Infantil; Isolamento Social; Crianças; Violência Infantil.

Os critérios de inclusão foram: serem documentos nacionais e internacionais, entre eles artigos, manuais, notas e cartilhas, que deveriam estar disponíveis na internet em língua portuguesa, inglesa e espanhola a partir das bases de dados pesquisadas com textos disponíveis na íntegra e gratuitos. Já os critérios de exclusão foram removidos, estudos que incluem como público-alvo outras fases de desenvolvimento (jovens-adultos, adultos e/ou idosos), teses, dissertações e monografias ou estudos que fugissem da área de pesquisa.

Após a busca nas três bases de dados, foram selecionados 90 conteúdos pelo título e resumo, destes foram excluídos 50, pois estavam duplicados ou fugiam do tema. Assim como, foram lidos e analisados na íntegra, 40 estudos que correspondiam aos critérios de inclusão da pesquisa. E destes emergiram um total de 12 achados que serão apresentados na seção resultados. E por fim, as análises temáticas foram construídas a partir da análise de conteúdo, que apresenta uma sequência: organização, interpretação e categorização dos resultados (Bardin, 2016).

\section{Resultados}

Os estudos que correspondem a esta revisão de literatura integrativa serão apresentados na Figura 1, por meio de um fluxograma de seleção dos materiais conforme os critérios de inclusão e exclusão. Quanto às características gerais dos documentos pesquisados, verificou-se que $100 \%$ (12) dos materiais correspondem ao ano de 2020 . Cerca de $25 \%$ (3) dos achados são documentos internacionais e 75\% (9) nacionais. Além disso, 41,6\% (5) dos materiais são artigos científicos publicados em periódicos e $58,3 \%$ (7) são outros materiais como cartilhas, manuais e notas de alerta. 
Figura 1- Fluxograma do processo de seleção dos estudos.

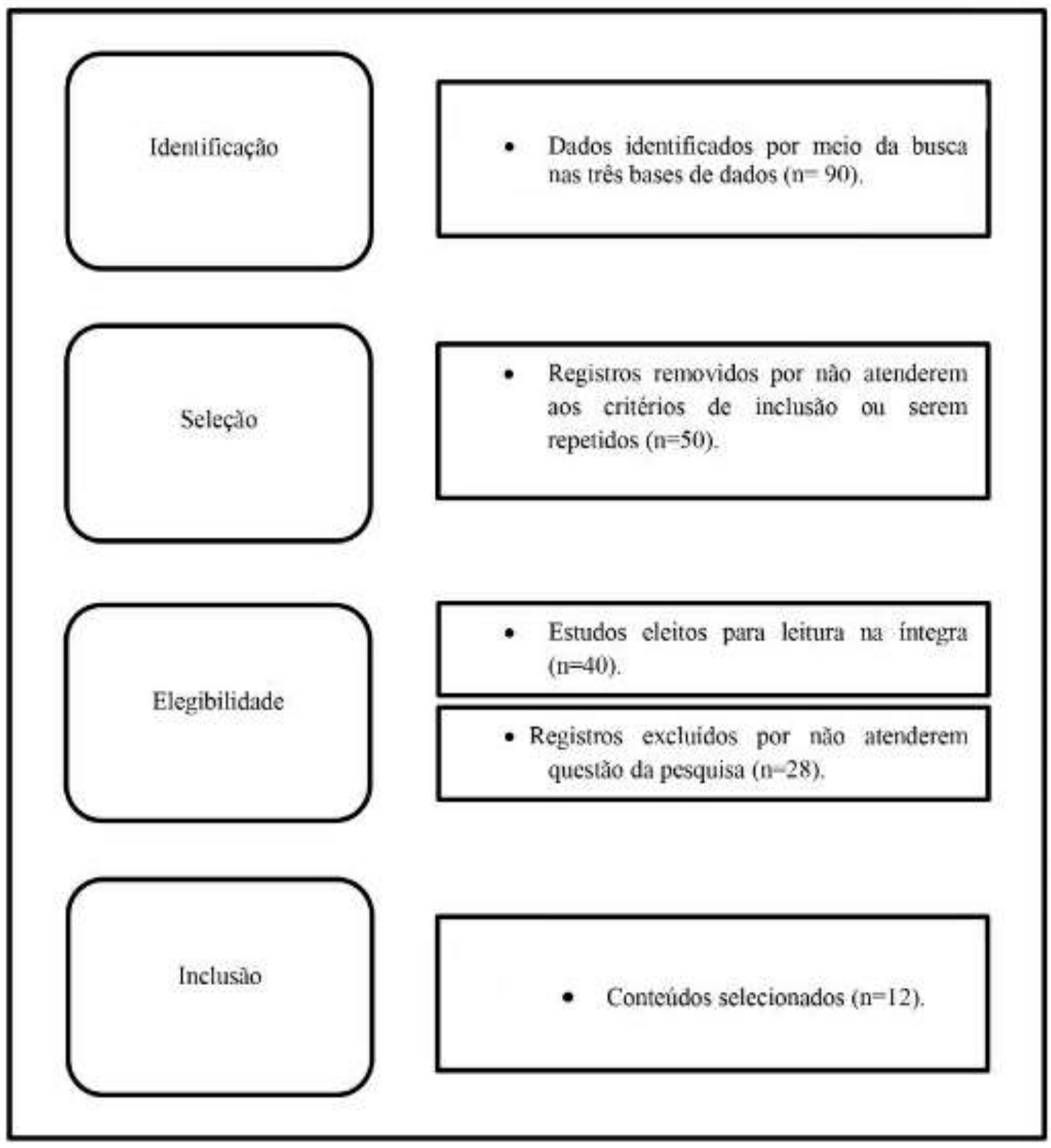

Fonte: Autores (2020).

A síntese dos materiais analisados encontra-se na Tabela 1, como a autoria, objetivos dos documentos e demais informações. Tendo em vista o objetivo deste estudo, compreender como as crianças estão vivenciando os impactos da pandemia e as suas repercussões no desenvolvimento infantil, extraíram-se quatro categorias: Tecnologia e saúde mental envolvendo as crianças durante a pandemia da COVID-19; A importância dos pais falarem sobre o processo de morte e morrer com os filhos; A violência infantil no contexto da COVID-19 e Informações para manter a saúde mental das crianças. 
Tabela 1 - Caracterização dos materiais selecionados para o estudo.

\begin{tabular}{llll}
\hline Referências & Objetivos & Tipo de documento & Ano
\end{tabular}

Barbancho, M.M.M., Apresentar um guia para

Cartilha

2020

Jiménez, A.C., Silva, A. G., familiares sobre $\mathrm{o}$ enfrentamento da morte, \& Viólas, L.C. como forma de aliviar a dor daquelas pessoas que estão passando por isso neste momento.

Crepaldi, M.A., Schmidt, Sistematizar conhecimentos Artigo B., Noal, D da S., Bolze, sobre os processos de D.A.S., \& Gabarra, L. M. terminalidade, morte e luto no contexto da pandemia de COVID-19.

Cruz, M. M.

Informar cientificamente o

Cartilha que é a COVID-19, por meio de uma ferramenta lúdica.

Eisenstein, E., Pfeiffer, L., Ensinar às crianças sobre as Gama, M. C., Estefenon, S., habilidades necessárias para \& Cavalcanti, S. S.

navegar com segurança e podem ser acessados com vários tópicos sobre cidadania digital.

Espada, J. P., Orgiléz, M., Conhecer os efeitos da Artigo Piquera, J. Á., \& Morales, A. quarentena a médio ou longo prazo, estudos têm sido realizados sobre a prevalência de estresse agudo ou pós-traumático em crianças e adolescentes.

Fundação Oswaldo Cruz. Apresentar aspectos sobre a

Cartilha saúde mental e atenção psicossocial de crianças no contexto da pandemia, destacando fatores relacionados à sobrecarga de trabalho e demandas no âmbito familiar.

Fundação Oswaldo Cruz. Oferecer subsídio aos

Cartilha

$2020 b$ profissionais da rede de proteção e cuidado às pessoas em situação de violência, bem como aos gestores e a todos os envolvidos na resposta à COVID-19, reforçando as 


\begin{tabular}{|c|c|c|c|}
\hline & $\begin{array}{l}\text { ações voltadas às pessoas em } \\
\text { situação de violência } \\
\text { doméstica e familiar durante } \\
\text { a crise. }\end{array}$ & & \\
\hline Fundação Oswaldo Cruz. & $\begin{array}{l}\text { Favorecer a adaptação aos } \\
\text { desafios impostos na } \\
\text { vigência das medidas de } \\
\text { distanciamento social, a } \\
\text { seguir são elencadas algumas } \\
\text { estratégias que podem ser } \\
\text { adotadas conforme } \\
\text { necessidades individuais e } \\
\text { coletivas. }\end{array}$ & Cartilha & $2020 c$ \\
\hline Maranhão, R. A. & $\begin{array}{l}\text { Investigar a violência contra } \\
\text { a mulher durante o período } \\
\text { de quarentena do COVID-19. }\end{array}$ & Artigo & 2020 \\
\hline $\begin{array}{l}\text { Marques, E. S, Moraes, C. } \\
\text { L. D., Hasselmann, M. H., } \\
\text { Deslandes, F. S., \& } \\
\text { Reichenheim, M.H. }\end{array}$ & $\begin{array}{l}\text { Discutir a violência contra } \\
\text { mulheres, crianças e } \\
\text { adolescentes em tempos de } \\
\text { pandemia. }\end{array}$ & Artigo & 2020 \\
\hline $\begin{array}{l}\text { Sociedade Brasileira de } \\
\text { Pediatria. }\end{array}$ & $\begin{array}{l}\text { Fornecer algumas dicas } \\
\text { importantes para os pais } \\
\text { nesse momento de pandemia. }\end{array}$ & Nota de alerta & 2020 \\
\hline $\begin{array}{l}\text { Wallace, C. L.W., } \\
\text { Wladkowski, S. P., Gibson, } \\
\text { A., \& White P. }\end{array}$ & $\begin{array}{l}\text { Discutir o combate da dor } \\
\text { através da comunicação, } \\
\text { planejamento antecipado de } \\
\text { cuidados e práticas de } \\
\text { autocuidado. }\end{array}$ & Artigo & 2020 \\
\hline
\end{tabular}

Fonte: Autores (2020).

\section{Discussões}

\section{Tecnologia e saúde mental envolvendo as crianças durante a pandemia da COVID-19}

Neste contexto de pandemia, houve uma mudança na rotina das famílias, sendo que muitos pais precisam trabalhar no esquema de home office, além disso precisam fazer as atividades relacionadas aos afazeres da casa, as compras, lidar com as saídas restritas, com as preocupações financeiras, com as informações sobre a pandemia e com os cuidados das crianças, sendo que muitas permanecem em casa em tempo integral devido às atividades escolares remotas (SBP, 2020). Sem o apoio dos avós ou demais membros da família, em virtude do isolamento social, muitos pais estão tendo que administrar a rotina diária, de forma a conciliar suas atribuições do trabalho e os cuidados com os filhos.

Ainda, é preciso considerar que muitos pais perderam seus empregos e precisam lidar com as dificuldades financeiras que se assolaram neste contexto de pandemia. Desta forma, o estresse é elevado e diário, podendo ser prejudicial a todos os membros da família. As consequências em curto prazo podem incluir transtorno do sono, irritabilidade, piora da imunidade, medos, a médio e longo prazo, com maior prevalência de atrasos no desenvolvimento, transtornos de ansiedade, depressão, queda no rendimento escolar e estilo de vida pouco saudável na vida adulta (Fiocruz, 2020a; SBP, 2020). 
Nesse atual cenário, o uso das telas (TV, smartphones, tablets, computadores) tem sido um aliado importante na manutenção dos laços sociais e afetivos para aquelas crianças que apresentam condições para ter acesso a esse recurso (Fiocruz, 2020b). Crianças em idades precoces têm tido acesso a telefones celulares e smartphones, notebooks além dos computadores que são usados pelos pais, irmãos ou família, em casa, nas creches, em escolas ou mesmo em quaisquer outros lugares com o objetivo de fazer com que a "criança fique quietinha" (Einstein, Pfeiffer, Gama, Estefenon, \& Cavalcanti, 2019). Cabe ressaltar que, embora o acesso pelas crianças aos meios digitais seja para fins escolares e, também, para estar em contato com pessoas queridas, também são utilizados para entretenimento e lazer. Uma vez que, as crianças se encontram confinadas em seus lares, distantes de amigos, colegas e seus familiares, assim como das atividades de recreação e socialização.

Embora o tempo diante de telas precise ser observado, e seja necessário garantir a adequação e a qualidade do conteúdo, há de se ter mais flexibilidade em seu uso. O que se depreende das publicações é que está havendo uma maior flexibilização por parte dos pais, nesse novo cenário de pandemia, quanto ao acesso e tempo de uso, na tentativa de dar conta das demandas exigidas pelo trabalho bem como os afazeres domésticos (Fiocruz, 2020b).

O tempo excessivo ou abusivo do uso das Tecnologias de Informação e Comunicação (TIC) bem como os seus efeitos são motivos de preocupação para pais e professores. Várias são as pesquisas e evidências científicas (Einstein et al., 2019: Fiocruz, 2020b; SBP, 2020) sobre os benefícios e também sobre os prejuízos à saúde, quanto ao uso precoce, excessivo e prolongado das tecnologias durante a infância e os efeitos em longo prazo. As recomendações oferecem parâmetros para o uso de telas fora das situações de crise, indicando que o tempo diário seja adequado à idade da criança, devendo-se desencorajar a exposição passiva (SBP, 2020).

Com relação à saúde mental de crianças na era digital, duas das publicações incluem a detecção de problemas como o Transtorno do déficit de atenção e hiperatividade (TDAH), irritabilidade, ansiedade e depressão, mesmo antes da pandemia neste artigo considerada (Einstein et al., 2019; SBP, 2020). Os principais fatores de risco apontados que estão relacionados a tais problemas foram a falta de afeto e abandono, falta de limites, negação dos comportamentos inadequados do/s filho/s, violência familiar, família disfuncional, episódios frequentes de eventos estressores crônicos, uso de álcool e drogas, falta de suporte e apoio. Por outro lado, os fatores de proteção são o diálogo e respeito, regras claras de convivência, modelos referenciais e saudáveis de identificação, olhar de cuidados, oportunidades e alternativas mais saudáveis, desenvolvimento de valores éticos, o não uso de álcool e drogas e apoio e resiliência familiar (Einstein et al., 2019; Espada, Orgiléz, Piquera, \& Morales, 2020).

Assim, neste cenário atual de pandemia, muito embora haja dificuldades encontradas pelos pais em conciliar as diversas demandas diárias, é imprescindível que o uso da tecnologia pelas crianças que possuem o acesso seja feita sob supervisão e de acordo com a faixa etária, evitando o acesso a conteúdos inadequados e o uso excessivo e prolongado com intuito de evitar possíveis prejuízos no desenvolvimento infantil (SBP, 2020). É válido destacar que vivemos em uma sociedade bastante discrepante, onde nem todas as pessoas possuem o acesso a esses dispositivos, pois quando se aborda sobre o uso ilimitado dos meios tecnológicos enfatizam-se as classes sociais mais favorecidas socialmente.

\section{A importância dos pais falarem sobre o processo de morte e morrer com os filhos}

A pandemia pela COVID-19 tem acarretado às pessoas perdas significativas de seus familiares e amigos. O luto é um processo de elaboração de perdas vividas que é experienciado de forma singular, e pode ser muito difícil e doloroso para quem o experimenta. As crianças pequenas podem viver a experiência da morte, no entanto não compreendem sua irreversibilidade e o que pode acontecer com pessoas queridas. As mais velhas, ainda que compreendam cognitivamente o significado da morte, podem ter poucos recursos psicológicos para lidar com a situação (Espada et al., 2020). 
A temática morte é um tabu, embora a finitude seja um processo natural e comum a todos os indivíduos, dialogar, mesmo entre os adultos, sobre esse assunto pode gerar medo, insegurança, temor e angústia. Muitas vezes, os adultos evitam falar sobre o processo de morte e morrer por acreditarem que as crianças não têm um preparo emocional necessário para o processamento e entendimento desse tema. Diante dos questionamentos da criança sobre a morte, muitos pais não sabem como se comportar e se sentem desconfortáveis e ansiosos, evitando dar respostas às perguntas dos seus filhos ou até mesmo dando uma explicação sem sentido (Yamaura \& Veronez, 2016).

Posto isso, as crianças passam pelo mesmo processo de luto e necessitam de esclarecimento e acolhimento dos seus sentimentos conforme seu momento desenvolvimental (Kovács, 2016). Elas podem experienciar o adoecimento de um familiar com profundo estresse, podendo afetar a sua saúde mental (Espada et al., 2020).

Frente ao adoecimento ou até mesmo à morte, muitos pais podem ficar na dúvida sobre contar ou não a verdade. $\mathrm{O}$ medo da exposição das crianças a momentos de tristeza pode ser prejudicial para que elas desenvolvam as habilidades emocionais que são necessárias para toda a vida. Devido à convivência ter aumentado, as crianças sentem que algo diferente do cotidiano está acontecendo, as preocupações ou dores dos pais são percebidas pelos filhos. Nesses casos, esconder a verdade ou mentir pode acabar até na perda de confiança das crianças (Barbancho, Jiménez, Silva, \& Viálas, 2020). Para os pais, ouvir e responder perguntas relacionadas a essa temática pode ser uma tarefa difícil, desta forma tentam evitá-la, afirmando que a criança vai sofrer ou não entender. A melhor maneira de lidar com o sofrimento é favorecer a conversa, compartilhando os sentimentos (Kovács, 2016).

Por outro lado, o adoecimento e a hospitalização pela COVID-19 podem ser vivenciados pela criança. Caso isso aconteça, deve estar acompanhada de um adulto de sua confiança, pois do contrário esse período de internação pode ser marcado pela agitação, crises de angústias e até mesmo o desenvolvimento da depressão. Outro ponto fundamental nesse caso é o relato verdadeiro da situação e do tratamento da criança, para que ela entenda e venha a se sentir segura diante do contexto (Fiocruz, 2020b).

De uma forma geral, na literatura é sugerido que os pais precisam ser honestos e francos, e principalmente, aberto para o esclarecimento de dúvidas que as crianças possam ter em relação à perda e morte. É necessária uma escuta atenta e respeitosa, mas que, acima de tudo, os pais ou responsáveis utilizem uma linguagem acessível para responder às dúvidas das crianças. É interessante que pais e filhos construam junto uma imagem carinhosa da pessoa que faleceu isso pode ajudar na elaboração do luto da criança (Yamaura \& Veronez, 2016).

A Psicologia costuma contribuir muito para que a criança expresse seus medos e anseios diante da perda e da morte. A ludoterapia utiliza-se do brincar e do desenhar das crianças como uma técnica para acessar seus sentimentos. Até mesmo as histórias de livros infantis, que envolvam a temática de morte podem facilitar no processo de identificação e aceitação da morte e do morrer (Wallace, Wladkowski, Gibson, \& White, 2020). Por fím, cabe ressaltar a importância do psicólogo no atendimento da criança e da família após a perda.

\section{A violência infantil no contexto da COVID-19}

A família tende a modificar toda a sua rotina em função do trabalho e do cuidado com as crianças (Fiocruz, 2020a). Neste período de pandemia os episódios de violência contra a criança e adolescentes podem se tornarem mais frequentes, há um aumento no tempo de convivência, bem como das tensões nas relações interpessoais. Além disso, é importante considerar que a capacidade de lidar com conflitos e a supervisão parental podem estar diminuídas em função de doenças mentais preexistentes e sua possibilidade de agravamento (Marques, Moraes, Hasselmann, Deslandes, \& Reichenheim, 2020). A permanência em casa pode ser exaustiva para toda a família, e os filhos muitas vezes tendem a querer a companhia dos pais a 
todo o momento, impossibilitando esses adultos de darem sequência nas suas atividades o que pode gerar estresse e esgotamento dos pais (Fiocruz, 2020b).

Pode-se pensar no aumento de violência como uma soma de fatores que o cenário vem proporcionando, como o afastamento social que implica no distanciamento de pessoas importantes para pais e filhos, o impacto negativo das finanças que estressam os pais, o aumento do uso de drogas e álcool, que impossibilita muitas vezes o adulto a pensar antes de agir (Fiocruz, 2020c). Ademais a quarentena assim como qualquer momento de crise intensifica sentimentos como o poder, o desejo, a dominação, o ódio, o estresse, a obediência e até mesmo a submissão (Maranhão, 2020). Indo ao encontro do que foi exposto, existem evidências e estudos que comprovam que a violência em crianças aumentou, bem como em adolescentes e mulheres (Marques et al., 2020).

Visto isso, a violência infantil é um tema que percorre a história da humanidade e a cada ano que passa tem aumentado seus níveis (Rates, Melo, Mascarenhas, \& Malta, 2015). Pode-se caracterizar a violência, em linhas gerais, como um ato ou atitude violenta ao utilizar da força física, psicológica ou até mesmo o poder contra si ou contra o outro causando dano psicológico, lesões, deficiência de desenvolvimento ou privação e até mesmo a morte (Nunes \& Sales, 2016).

Ao tentarmos visualizar essa problemática em dados, uma pesquisa realizada em 96 países denuncia que mais de 50\% (cinquenta por cento) das crianças brasileiras sofrem ou já haviam sofrido algum tipo de violência, seja ela física, sexual ou psicológica. Ademais, a violência na maioria das vezes é escondida tanto por quem pratica, quanto por quem sofre (OMS, 2018).

A maioria dos casos de violência independente de classe social é cometida por aquelas pessoas que deveriam proteger as crianças na condição de pais ou responsáveis. Os dados apontam que a faixa etária mais acometida por esses abusos são crianças de cinco a 12 anos. No caso das meninas a violência na maioria das vezes é sexual, e no caso dos meninos a violência geralmente é física (Nunes \& Sales, 2016).

As causas que tentam justificar a violência são inúmeras, desde uma ordem individual, que diz respeito à educação, a renda, o histórico do comportamento, como também a nível social, que se referem a práticas parentais inadequadas, casamento forçado, dentre outros tantos. No entanto, cabe ressaltar que em situações de crise, como guerras, desastres ou em surtos de doenças, a tendência é que ocorra um aumento com relação à violência (OMS, 2018).

Cabe ressaltar que algumas crianças, em virtude do contexto de isolamento social, estão sujeitas a terem acesso mais fácil e a passarem por mais tempo envolvidas nas redes sociais, o que as tornam alvos fáceis para pedófilos ou pessoas malintencionadas. Desta forma, a supervisão dos pais, quanto ao tempo e quais os conteúdos estão sendo acessados, pela criança é fundamental para evitar esse tipo de violência (Fiocruz, 2020c). Algumas vezes a supervisão parental pode ser impossibilitada pelo adoecimento de alguém próximo (Marques et al., 2020).

Para minimizar essas mazelas, inúmeras ações estão sendo tomadas, entre elas as criações de cartilhas, propagação de números de disque denúncia e propagandas de conscientização (Fiocruz, 2020c). Dois anos antes da pandemia já havia criado uma cartilha com sete estratégias para erradicar a violência infantil, entre elas: implementação e vigilância do cumprimento das leis que protegem contra a violência, modificação de normas e valores a fim de promover atitudes não violentas, promover a segurança do ambiente da criança, dar apoio à mãe, pais e cuidadores para que a violência aos poucos seja extinta da vida da criança, incrementar a renda e o fortalecimento econômico, melhorar os serviços de atendimentos a saúde, educar e ensinar habilidades para a vida (OMS, 2018).

Por fim, essas estratégias citadas acima são em longo prazo. No momento, o que se pode fazer, para defender as crianças que estão em situações de violência, é reforçar atitudes governamentais ou não governamentais que tenham essa finalidade de proteção. A garantia do atendimento vinte a quatro horas de serviços de disque-denúncia é fundamental. No caso 
das crianças pode-se enfatizar a importância do atendimento pelo Conselho Tutelar via WhatsApp, telefone ou outros meios digitais.

\section{Informações para manter a saúde mental das crianças}

Diante do cenário atual, as rotinas familiares necessitam ser adaptadas devido às medidas de distanciamento. Contudo, muitas famílias não estão conseguindo se organizar e dar conta de todas as demandas implicadas nesse novo contexto, pois os filhos não estão na escola, o trabalho em muitas famílias incorpora a modalidade home office e os afazeres de casa tendem a aumentar (SBP, 2020).

Todo esse excesso pode gerar impactos negativos no campo da saúde mental das crianças. O estresse, raiva, ansiedade e agressão já foram estudados e comprovados em outras situações prolongadas de isolamento. Para minimizar esses efeitos negativos enfatiza-se a importância do cuidado da saúde mental como uma forma de prevenção de problemas mentais futuros (Jeong et al., 2016).

Nesse sentido, torna-se fundamental estabelecer uma rotina familiar, mas é importante ressaltar que cada família deve instituir a sua organização frente à rotina, não existe um modelo fixo e igualitário a todos os contextos familiares, porque cada ambiente possui realidades muito particulares. No entanto, estipular um planejamento em relação a uma boa alimentação, períodos do dia para momentos de lazer e de interação com as crianças, assim como horários prévios para adormecer se fazem essenciais para a dinâmica dos membros da família (Fava, Rosa, \& Olivia, 2018).

O planejamento e a execução da rotina minimizam a chance do estresse. A inclusão de exercícios físicos nas atividades diárias pode diminuir a probabilidade de ansiedade e depressão. Aproveitar o tempo para uma leitura ou realizar um curso on-line também são pontos positivos para a cognição dos cuidadores de crianças. Deve-se ressaltar a importância da convivência familiar, escutar, brincar e se incluir no universo das crianças. Tudo isso é importante para que os filhos não se sintam solitários, os pais precisam prestar atenção nas suas emoções, bem como nos sentimentos que a criança expressa diante da realidade vivida (Fiocruz, 2020b).

Ainda sobre as estratégias que os pais podem utilizar para manter a saúde mental dos filhos, destaca-se a importância de os adultos conversarem e trocarem informações sobre como tornar esse período menos estressante para a criança. Em alguns casos pode ocorrer do filho que não está acostumado com a presença materna e paterna em casa, exigir toda a atenção e o tempo desse adulto. Tudo isso pode provocar momentos de tensão e raiva, pois os pais tendem não conseguir efetuar suas tarefas relativas ao trabalho. Nesse caso, torna-se essencial dialogar com a criança, e estabelecer combinados em relação aos horários de diversão com os filhos (SBP, 2020).

Devido a impossibilidade da criança estar com seus pares ou mesmo encontrar e receber visitas de pessoas que são importantes para ela, todo esse confinamento pode acabar gerando sentimento de tristeza e impotência. É necessário que os pais compartilhem e aceitem esses sentimentos, da mesma forma que tentam diminuir a distância social utilizando a tecnologia como um fator positivo. Embora o abraço, o beijo e a presença sejam alternativas temporariamente dispensadas, a distância pode ser minimizada com recursos de vídeo chamada ou ligações telefônicas. A escrita também pode ser uma dica para que as crianças e adultos se tranquilizem diante da pandemia. Escrever sozinho ou com o filho, além de resolver conflitos interiores pode acabar por gerar uma união familiar ainda maior (Barbancho et al., 2020).

Neste contexto de distanciamento social, quando aplicado devido à pandemia da COVID-19, há necessidade de empregarem-se as práticas baseadas em evidências científicas, que podem contribuir na preservação do bem estar das crianças e do grupo familiar. Entre essas, inclui-se a definição com as crianças dos horários para o uso saudável das telas, evitando ultrapassar os limites e o acesso sem supervisão a conteúdos inadequados, além de definir horários para jogos on-line com os amigos e para videoconferências com os avós (SBP, 2020). 
Contudo, é notório que sites, programas de televisão e noticiários perante o cenário do coronavírus no Brasil e no mundo acabam despejando, principalmente, notícias infelizes e angustiantes. A morte é transmitida pela mídia para as crianças com cenas chocantes, deprimidas e com textos superficiais (Crepaldi et al., 2020; Kovacs, 2016). Nesse sentido, se faz necessário um controle dos pais ao que as crianças acessam na internet, ou mesmo aos programas de televisão que assistem (Fiocruz, 2020b).

Outro ponto fundamental que a cartilha da Fundação Oswaldo Cruz (2020b) discute é referente ao diálogo sobre o momento que estamos passando com a criança. Tentar explicar de uma maneira honesta a realidade e utilizar do momento para educá-las quanto às práticas de higiene e de prevenção perante a contaminação do vírus. Nos casos em que a criança é muito pequena ou os pais apresentam dificuldades de explicar o panorama que o mundo vivencia, pode-se utilizar de diversos materiais que foram construídos de forma lúdica para o entendimento da criança (SBP, 2020).

Foi criado um documento ilustrativo e lúdico para as crianças, que busca explicar o que é o coronavírus, como ocorre sua transmissão e como se proteger (Cruz, 2020). É importante enfatizar que, nesse momento, os pais têm uma função importante na prevenção e promoção de saúde dos filhos.

\section{Considerações Finais}

Conclui-se, através da literatura encontrada, que as crianças podem estar vivenciando negativamente a pandemia e isso repercute no desenvolvimento infantil, uma vez que alguns pais estão trabalhando na modalidade de home office, e ainda precisam cuidar dos pequenos em tempo integral. Através da revisão integrativa observou-se que a tecnologia se faz mais presente que no período anterior à pandemia e isso inclui o homeschooling. Dessa forma as crianças passam mais tempo conectadas, e isso pode trazer consequências em curto prazo como prejuízo do sono, irritabilidade, piora da imunidade, medos, a médio e longo prazo, com maior prevalência de atrasos no desenvolvimento, transtornos de ansiedade, depressão, queda no rendimento escolar e estilo de vida pouco saudável na vida adulta.

Com relação à temática de adoecimento, morte, hospitalização pela COVID-19, as crianças podem experienciar com medo, estresse e tristeza todo esse período, principalmente se os pais não conversarem sobre o contexto que o Brasil e o mundo vivenciam. Destaca-se também que a violência infantil pode ter aumentado nesse período, seja através da força física ou por abusos psicológicos, principalmente no panorama da hiper convivência familiar, assim podemos encontrar crianças mais agitadas, irritadas e desobedientes, além de afetar em todo desenvolvimento.

Por fim, salienta-se que a principal limitação devido ao contexto pandêmico está relacionada ao recorte social, já que as crianças do estudo referem-se possivelmente a uma classe econômica média/alta, que possuem todo um aparato tecnológico. Ainda existem poucas pesquisas que se aprofundaram nas temáticas apresentadas neste artigo. Dessa forma, sugere-se que sejam realizadas pesquisas futuras a fim de entender as repercussões no desenvolvimento infantil daquelas crianças que não conseguiram acessar ou manter-se nas aulas remotas, por exemplo. Também se sugere que seja foco de investigação científica as famílias que tiveram mudanças significativas, tal como perda de emprego dos pais, declínio financeiro considerável ou morte de entes queridos, entre outros.

\section{Referências}

Barbancho, M. M. M., Jiménez, A. C., Silva, A. G., \& Viólas, L. C. (2020). Covid-19: Guía para familiares en duelo. Colégio Oficial de Psicologia de Andalucia Occidental, 4(24). http://www.infocoponline.es/pdf/GUIA_PARA_FAMILIARES_EN\%20DUELO_GT\%20Duelo.pdf

Bardin, L. (2016). Análise de conteúdo. São Paulo: Edições 70.

Centro de estudos e pesquisas em emergências e desastres humanos. Fundação Oswaldo Cruz. (2020a). Saúde mental e Atenção Psicossocial na Pandemia COVID-19: A quarentena na COVID-19: orientações e estratégias de cuidado. https://www.fiocruzbrasilia.fiocruz.br/wp- 
content/uploads/2020/04/Sa\%c3\%bade-Mental-e-Aten\%c3\%a7\%c3\%a3o-Psicossocial-na-Pandemia-Covid-19-A-quarentena-na-Covid-19orienta\%c3\%a7\%c3\%b5es-e-estrat\%c3\%a9gias-de-cuidado.pdf

Centro de estudos e pesquisas em emergências e desastres humanos. Fundação Oswaldo Cruz. (2020b). Saúde mental e Atenção Psicossocial na Pandemia COVID-19: Crianças na Pandemia COVID-19. https://www.fiocruzbrasilia.fiocruz.br/criancas-e-pandemia-como-abordar-as-reacoes-emocionais-ecomportamentais/

Centro de estudos e pesquisas em emergências e desastres humanos. Fundação Oswaldo Cruz. (2020c). Saúde mental e Atenção Psicossocial na Pandemia COVID-19: Violência Doméstica e Familiar na COVID-19. https://www.arca.fiocruz.br/bitstream/icict/41121/2/Sa\%c3\%bade-Mental-eAten\%c3\%a7\%c3\%a3o-Psicossocial-na-Pandemia-Covid-19-viol\%c3\%aancia-dom\%c3\%a9stica-e-familiar-na-Covid-19.pdf

Crepaldi, M. A, Schmidt, B. , Noal, D. da S. , Bolze, S. D. A., \& Gabarra, L. M. (2020). Terminalidade, morte e luto na pandemia de COVID-19: demandas psicológicas emergentes e implicações práticas. Estudos de Psicologia (Campinas), 37, e200090. Epub June 01, 2020. doi: 10.1590/1982-0275202037e200090

Cruz, M. M. (2020). Olá, sou um vírus, primo da gripe e do resfriado. Recuperado de www.mindheart.co/descargables.

Espada, J. P., Orgiléz, M., Piquera, J. A., \& Morales, A. (2020). Las Buenas Prácticas en Lá Atención Psicológica Infanto-Juvenil ante ella COVID-19. Clínica y Salud. https://journals.copmadrid.org/clysa/art/clysa2020a14

Eisenstein, E., Pfeiffer, L., Gama, M. C., Estefenon, S., \& Cavalcanti, S. S. (2019). Manual de orientação: \#Menos telas\# Mais saúde. Sociedade Brasileira de Pediatria. Recuperado de https://www.sbp.com.br/fileadmin/user_upload/_22246c-ManOrient_-__MenosTelas__MaisSaude.pdf

Fava, D. C., Rosa, M., \& Olivia, A.D. (2018). Orientação para Pais. O que é preciso saber para cuidar de um filho. Belo Horizonte: Artesã.

Jeong, H., Yam H. W., Song, Y. J., Ki, M., Min, J. A., Sho, J., \& Chae, J. H. (2016). Mental health status of people isolated due to Middle East Respiratory Syndrome. Epidemyology and Health, 38(5). doi: 10.4178/epih.e2016048

Kovacs, M. J. (2016). Falando de morte com as crianças. Psico USP, 2(3). Recuperado de http://www.ip.usp.br/revistapsico.usp/index.php/30-commentor2/79-falando-de-morte-com-criancas.html

Maranhão, R. A. (2020). A violência doméstica durante a quarentena da COVID-19: entre romances, feminicídios e prevenção. Brazilian Journal of health Review, 3 (2), 3197-3211. doi: 10.34119/bjhrv3n2-161

Marques, E. S., Moraes, C. L. D., Hasselmann, M. H., Deslandes, F. S., \& Reichenheim, M. H. (2020). A violência contra mulheres, crianças e adolescentes em tempos de pandemia pela COVID-19: panorama, motivações e formas de enfrentamento. Cadernos de Saúde Pública, 36(4), 1-6. doi: 10.1590/0102$311 \mathrm{X} 00074420$

Nunes, A. J., \& Sales, M. C. V. (2016). Violência contra crianças no cenário brasileiro. Ciência \& Saúde Coletiva, 21(3),871-880. doi: 10.1590/141381232015213.08182014

Organização Pan-Americana de Saúde [OPAS] (Brasil). (2020). OMS afirma que CONVID é agora caracterizada como Pandemia. Recuperado de https://www.paho.org/bra/index.php?option=com_content\&view=article\&id=6120:oms-afirma-que-covid-19-e-agora-caracterizada-comopandemia\&Itemid $=812$

Organização Mundial da Saúde. (2018). Cartilha Inspire: sete estratégias para pôr fim à Violência contra crianças. https://apps.who.int/iris/bitstream/handle/10665/207717/9789241565356-por.pdf?ua=1

Rates, S. M. M., Melo, E. M. D., Mascarenhas, M. D. M., \& Malta, D. C. (2015). Violence Against children: an analysis of mandatory reporting of violence, Brasil 2011. Ciência saúde coletiva, 20 (3), 655-666. doi: 10.1590/1413-81232015203.15242014

Rodrigues, R. M. (2018). Solidão, um fator de risco. Revista Portuguesa de Medicina Geral e Familiar, 34(5), 334-338. doi: 10.32385/rpmgf.v34i5.12073

Silva, C. C. D., Savian, C. M., Prevedello, B. P., Zamberlan, C., Dalpian, D. M., \& Santos, B. Z. D. (2020). Access and use of dental services by pregnant women: an integrative literature review. Ciência \& Saúde Coletiva, 25, 827-835. doi: 10.1590/1413-81232020253.01192018

Sociedade Brasileira de Pediatria. Pais e filhos em confinamento durante a pandemia de Covid-19. (2020). Departamento Científico de Pediatria do desenvolvimento e comportamento. Recuperado de https://www.sbp.com.br/imprensa/detalhe/nid/pais-e-filhos-em-confinamento-durante-a-pandemia-decovid-19/

Yamura, L. M. P., \& Veronez, F. D. S. (2016). Comunicação sobre a morte para as crianças: estratégia de intervenção. Psicologia Hospitalar, 14 (1), 79-93. http://pepsic.bvsalud.org/pdf/ph/v14n1/14n1a05.pdf

Wallace, C. L. W., Wladkowski, S. P. Gibson, A., \& White P. (2020). Grief During the COVID-19 Pandemic: Considerations for Palliative Care Providers. Journal of Pain and Sympton management. doi: 10.1016/j.jpainsymman.2020.04.012

Zimmermann, P., \& Curtis, N. (2020). Coronavirus in Clidren including COVID-19: An overview of the epidemiology, Clinical Fatures, diagnostic, Treatament and Prevention options in Children. The Pediatric Doases journal, 12(5), 355-368. https://journals.lww.com/pidj/Fulltext/2020/05000/Coronavirus_Infections_in_Children_Including.1.aspx 